\title{
Necessary and sufficient condition for quantum state-independent contextuality
}

\author{
Adán Cabello, ${ }^{1, *}$ Matthias Kleinmann, ${ }^{2, \dagger}$ and Costantino Budroni ${ }^{3,}$ \\ ${ }^{1}$ Departamento de Física Aplicada II, Universidad de Sevilla, E-41012 Sevilla, Spain \\ ${ }^{2}$ Department of Theoretical Physics, University of the Basque Country UPV/EHU, P.O. Box 644, E-48080 Bilbao, Spain \\ ${ }^{3}$ Naturwissenschaftlich-Technische Fakultät, Universität Siegen, Walter-Flex-Str. 3, D-57068 Siegen, Germany
}

(Dated: April 12, 2018)

\begin{abstract}
We solve the problem of whether a set of quantum tests reveals state-independent contextuality and use this result to identify the simplest set of the minimal dimension. We also show that identifying state-independent contextuality graphs [R. Ramanathan and P. Horodecki, Phys. Rev. Lett. 112, 040404 (2014)] is not sufficient for revealing state-independent contextuality.
\end{abstract}

PACS numbers: 03.65.Ud, 03.65.Ta

Introduction. - Contextuality, i.e., that the result of a measurement does not reveal a preexisting value that is independent of the set of comeasurable measurements jointly realized (i.e., the context of the measurement), is one of the most striking features of quantum theory and has been recently identified as a critical resource for quantum computing [1-3]. The earliest manifestation of contextuality in quantum theory is the Kochen-Specker theorem $[4,5]$, which states that, if the dimension $d$ of the quantum system is greater than 2 , there exists a finite set of elementary tests (represented by rank-one projectors in quantum theory) such that a value 1 or 0 (representing true or false, respectively) cannot be assigned to each of them respecting that: (i) result 1 cannot be assigned to two mutually exclusive tests (represented in quantum theory by mutually orthogonal projectors), and (ii) result 1 must be assigned to exactly one of $d$ mutually exclusive tests. Sets of elementary tests in which this assignment is impossible are called Kochen-Specker sets [6].

Assumptions (i) and (ii) are not needed for detecting contextuality. It can be revealed by the violation of correlation inequalities satisfied by any model with noncontextual results. These inequalities are called noncontextuality (NC) inequalities [7]. Bell inequalities [8] are a special case of them.

Remarkably, there are NC inequalities which are violated by any quantum state for a fixed set of measurements [9]. A NC inequality with this property is called a state-independent $N C(S I-N C)$ inequality, whereas a set of elementary tests which can be used for such a stateindependent violation is called a state-independent contextuality (SIC) set.

Every Kochen-Specker set is a SIC set [10, 11], but there are SIC sets that are not Kochen-Specker sets $[12,13]$. This observation, together with the experimental implementation of SIC sets for testing SI-NC inequalities [14-19] and the emergence of applications of SIC sets (e.g., device-independent secure communication [20], local contextuality-based nonlocality [21], Bell inequalities revealing full nonlocality [22], state-independent quantum dimension witnessing [23], and state-independent hardware certification [24]) stimulated the interest in the problem of identifying SIC sets.

In some cases, one can guess that a given set of elementary quantum tests is a SIC set. Then, to prove it, it is sufficient to construct a SI-NC inequality violated by these tests. For example, the set of elementary quantum tests associated with the Peres-Mermin square [25, 26] violates a SI-NC inequality [9]; therefore, it is a SIC set. However, in general, one cannot follow this strategy and it is convenient to adopt a more general point of view and consider not a specific set of elementary quantum tests, but all sets of elementary quantum tests with a given exclusivity graph. In this graph, vertices correspond to tests and edges occur when two tests are mutually exclusive. Since elementary tests are represented by rankone projectors and two of them are mutually exclusive if and only if the corresponding projectors are orthogonal, the exclusivity graph is equivalent to the orthogonality graph of the corresponding projectors. This approach using graphs has been very successful in investigating the general properties of quantum contextuality [27, 28] and the separation between quantum theory and other hypothetical theories [29-33]. An open question is when, for a given orthogonality graph, there exists a realization of the graph which is a SIC set. Unfortunately, it has been notoriously difficult to answer this question [34]. The aim of this Letter is to provide a versatile tool that allows one to approach this problem.

Recently, Ramanathan and Horodecki ( $\mathrm{RH}$ ) [35] have presented a solution to a relaxation of the problem of identifying SIC sets, namely of identifying "SIC graphs." That is, whether a given graph admits, for any given state, a realization as a set of projectors (with orthogonality relations corresponding to edges in the graph) such that the correlations of such projectors on that state violate some NC inequality. This definition fits neither with the definition of a SIC set above nor with most of the previous literature (cf. Refs. [9, 10, 12, 13, 21-24, 34]). As far as we know, the only work where a similar definition has been used is Ref. [36]. Moreover, the definition of a SIC set in Ref. [35] is not state independent on an operational level. The issue is that, according to this definition, the realization of a SIC graph may depend 
on the state; the set of measurements that violate the $\mathrm{NC}$ inequality may be different for different initial states. Therefore, the definition is not state independent on an operational level. To make an analogy, adopting a similar definition one will reach the conclusion that a pentagon is a "SIC graph for pure states" since any pure state will violate the Klyachko-Can-Binicioğlu-Shumovsky NC inequality [37] for some five rank-one projectors whose orthogonality graph is a pentagon. In contrast, the problem of identifying SIC sets not only has a long tradition (cf. Refs. [6, 12, 13]), but also an immediate experimental translation (cf. Refs. [17-19, 24]).

To prove that the result in Ref. [35] does not solve the problem of identifying SIC sets, we begin by showing that there exists a SIC graph for which no realization violates a NC inequality for every quantum state (Theorem 1). After that, we present a solution to the problem of identifying SIC sets (Theorem 3). Finally, we use it to prove a conjecture formulated by $\mathrm{Yu}$ and $\mathrm{Oh}$ in Ref. [12] on the simplest SIC set in $d=3$ (Theorem 5).

From graph theory we will use the notions of the chromatic number and the fractional chromatic number of a graph (cf. Ref. [38]). Given a graph $G$, i.e., a set of vertices and the edges connecting them, a coloring of the graph is an assignment of colors to vertices such that vertices connected by an edge are associated with different colors. The chromatic number $\chi(G)$ is the minimum number of colors needed. Similarly, the fractional chromatic number $\chi_{f}(G)$ is the minimum of $\frac{a}{b}$ such that vertices have $b$ associated colors, out of $a$ colors, where vertices connected by an edge have associated disjoint sets of colors. $\chi_{f}(G)$ can be computed as a linear program.

Results. - The operational state dependence of a SIC graph as defined in Ref. [35] is apparent in the following theorem.

Theorem 1. There exists a SIC graph for which no realization is a SIC set.

Proof. - In Ref. [35] it is proven that a necessary and sufficient condition for a graph $G$ with a $[d, r]$-realization (i.e., a realization in dimension $d$ by means of rank- $r$ projectors) to be a SIC graph is that the fractional chromatic number $\chi_{f}(G)$ is strictly larger than $d / r$.

However, consider the 13-vertex graph of $\mathrm{Yu}$ and $\mathrm{Oh}$ [12], $G_{Y O}$. This graph has a [3,1]-realization and its fractional chromatic number is $\chi_{f}\left(G_{\mathrm{YO}}\right)=\frac{35}{11}$. Now consider the 14-vertex graph $G_{\mathrm{YO}+1}$ constructed by adding one vertex to $G_{\mathrm{YO}}$ and linking this new vertex with the 13 vertices of $G_{\mathrm{YO}}$. Clearly, this graph has a $[4,1]$ realization and $\chi_{f}\left(G_{\mathrm{YO}+1}\right)=\frac{35}{11}+1>4$. It is true that, for any state in $d=4$, there is a realization which violates a $\mathrm{NC}$ inequality. However, whatever the realization, when the system is in the eigenstate corresponding to the new vertex, there is an obvious noncontextual assignment of results, namely, one to the 14 th projector and zero to all others.
Now we will address the problem of identifying SIC sets. We first recall a result from Ref. [39] that helps us to identify sets of (not necessarily rank-one) projectors for which there is a SI-NC inequality.

Theorem 2. A set of observables $\left\{A_{1}, \ldots, A_{n}\right\}$ with spectra $\left\{\sigma\left(A_{1}\right), \ldots, \sigma\left(A_{n}\right)\right\}$, and contexts $C$ (i.e., the set of sets of comeasurable observables) violates the SI-NC inequality

$$
\sum_{c \in C} \lambda_{c}\left\langle\prod_{k \in c} A_{k}\right\rangle \leq \eta
$$

with $0 \leq \eta<1$ and real coefficients $\lambda_{c}$, if and only if

$$
\sum_{c \in C} \lambda_{c} \prod_{k \in c} a_{k} \leq \eta \text { for all } a \text { and } \sum_{c \in C} \lambda_{c} \prod_{k \in c} A_{k} \geq \mathbb{1},
$$

where the entries $a_{k}$ in $a=\left(a_{1}, \ldots, a_{n}\right)$ assume any value from $\sigma\left(A_{k}\right)$.

Then, the necessary and sufficient condition for a set of rank-one projectors to constitute a SIC set is given by the following.

Theorem 3. A set of rank-one projectors $S=$ $\left\{\Pi_{1}, \ldots, \Pi_{n}\right\}$ is a SIC set if and only if there are nonnegative numbers $w=\left(w_{1}, w_{2}, \ldots\right)$ and a number $0 \leq$ $y<1$ such that

$$
\sum_{j \in \mathcal{I}} w_{j} \leq y \text { for all } \mathcal{I} \text { and } \sum_{i} w_{i} \Pi_{i} \geq \mathbb{1},
$$

where $\mathcal{I}$ is any set such that $i, j \in \mathcal{I}$ implies $\Pi_{i} \Pi_{j} \neq 0$ (i.e., $\mathcal{I}$ is any independent set of the orthogonality graph of $S)$.

In particular, $w$ gives rise to the SI-NC inequality

$$
\sum_{i} w_{i}\left\langle\Pi_{i}\right\rangle-\sum_{i} w_{i} \sum_{j \in \mathcal{N}(i)}\left\langle\Pi_{i} \Pi_{j}\right\rangle \leq y
$$

where $\mathcal{N}(i)=\left\{j \mid \Pi_{i} \Pi_{j}=0\right\}$ is the orthogonality neighborhood of $i$.

Proof.-For proving sufficiency, we will prove that, for a given $(y, w)$ satisfying conditions $(3)$, with $0 \leq y<1$, inequality (4) is a valid NC inequality and it is violated for every state. For that, it is enough to realize that among the noncontextual assignments maximizing the left-hand side of inequality (4) are those that respect the orthogonality conditions; i.e., two orthogonal projectors could not both have been assigned the value 1 . Respecting the orthogonality conditions precisely amounts to assign 1 to the elements of a set $\mathcal{I}$ appearing in conditions (3) and, hence, the bound $y$ holds for inequality (4). The proof goes as follows. Let us consider orthogonal projectors $\Pi_{i}$ and $\Pi_{j}$ and any noncontextual assignment $p \in\{0,1\}^{n}$ such that $p_{i}=1$ but $p_{j}=0$. By changing the value of $p_{j}$, i.e., violating the orthogonality 
condition, we get an extra contribution $w_{j}$ from the first term and $-\sum_{k \in \mathcal{N}(j)}\left(w_{j}+w_{k}\right) p_{k} \leq-w_{j}$ from the second term, decreasing the total value of the left-hand side of inequality (4). This proves that inequality (4) is a valid $\mathrm{NC}$ inequality. By condition (3), it is violated by any quantum state.

For proving necessity, we show that if $\left\{\Pi_{i}\right\}$ give rise to a violation of a linear $\mathrm{NC}$ inequality for every state, then conditions (3) are satisfied. Let us assume, for some $(\lambda, \eta)$, that the following inequality is violated by any state

$$
\sum_{\mathcal{C}} \lambda_{\mathcal{C}}\left\langle\prod_{k \in \mathcal{C}} \Pi_{k}\right\rangle \leq \eta
$$

where the sum is over all cliques $\mathcal{C}$ different from the empty set in the orthogonality graph of $S$, corresponding to all possible contexts, and $\lambda_{\mathcal{C}}$ are real numbers. Notice that the use of a linear expression in inequality (5) is not a restriction as it follows from the Hahn-Banach theorem (cf., e.g., Ref. [40]). In fact, the set of quantum correlations for all states and the set of noncontextual correlations are (compact) convex sets, and hence the sets either intersect or they can be separated by a hyperplane, i.e., distinguished via a linear inequality. Notice also that inequality (5) contains all of the possible correlations that are jointly measurable; i.e., it includes all contexts $\mathcal{C}$, with a generic coefficient $\lambda$.

Since inequality (5) holds, in particular, for all assignments respecting orthogonality, we have $\sum_{k \in \mathcal{I}} \lambda_{\{k\}} \leq \eta$ for any independent set $\mathcal{I}$. At the same time, we assume a state-independent violation and hence, without loss of generality, $\sum_{k} \lambda_{\{k\}} \Pi_{k} \geq \mathbb{1}$ and $\eta<1$. [In general we have $\sum_{k} \lambda_{\{k\}} \Pi_{k} \geq \xi \mathbb{1}$ and $\eta<\xi$. But the assignment $p \equiv(0,0, \ldots)$ yields $0 \leq \eta<\xi$, which allows us to rescale $\lambda_{\mathcal{C}} \rightarrow \lambda_{\mathcal{C}} / \xi$ and $\eta \rightarrow \eta / \xi$.] Eventually, we identify $w_{i}=\max \left\{0, \lambda_{\{i\}}\right\}$ and $y=\eta$. Indeed, inequality (5) has to hold for any assignment $p=\left(p_{1}, \ldots, p_{n}\right)$ respecting orthogonality and having $p_{k}=0$ for all $\lambda_{\{k\}}<0$. This way, the condition in Eq. (3) is obeyed by that identification.

We mention that the condition in Theorem 2 as well as that in Theorem 3 can be verified by means of a semidefinite program. Semidefinite programs are a class of optimization problems that can be solved numerically with a certificate of optimality [41].

At this point, it is interesting to point out the relation between Theorem 3 and the results in Ref. [35]. According to Ref. [35], to conclude that a graph of orthogonality is a SIC graph, it is sufficient to check the expectation value of $\sum_{j} w_{i} \Pi_{i}$ on the maximally mixed state $\rho=\mathbb{1} / d$. Assuming rank-one projectors, we can substitute the condition $\sum_{i} w_{i} \Pi_{i} \geq \mathbb{1}$ with $\frac{1}{d} \sum_{i} w_{i} \geq 1$, yielding RH's result. In fact, the condition in Eq. (3) can be formulated in terms of the existence of a solution greater than $d$ for the linear program

$$
\begin{aligned}
\text { maximize: } & \sum_{i} w_{i} \\
\text { subject to: } & \sum_{j \in \mathcal{I}} w_{j} \leq 1 \text { for all } \mathcal{I}, \\
& w_{i} \geq 0 \text { for all } i .
\end{aligned}
$$

Every $(w, y)$ obeying Eq. (3) with $y<1$ can be used to achieve $\sum_{i} w_{i}>d$ by rescaling all the weights by $1 / y$. The linear program in Eq. (6) is the dual problem of the fractional chromatic number $\chi_{f}(G)$ of the orthogonality graph $G$ (also known as the fractional clique number, cf. Ref. [38]); hence, both yield the same optimal value.

Together with the fact that the chromatic number $\chi(G)$ is lower bounded by the fractional chromatic number $\chi_{f}(G)$ [38], we have the following.

Theorem 4. Necessary conditions for a set of rank-one projectors in dimension $d$ to be a SIC set are that for the orthogonality graph $G$, (i) $\chi_{f}(G)>d$ and (ii) $\chi(G)>d$.

Condition (i) is also a direct consequence of the results in Ref. [35], where it was demonstrated in addition that, in general, condition (ii) is strictly weaker than condition (i). However, condition (ii) has the advantage of being solvable exactly by simple integer arithmetic, while condition (i) is the solution to a linear program.

The minimal dimension in which SIC sets exist is $d=3$ [5]. Therefore, identifying the smallest SIC set in $d=$ 3 is a problem of fundamental importance. Using the previous results we can prove a conjecture from Ref. [12].

Theorem 5. In dimension $d=3$, there exists no SIC set with less than 13 projectors. The set provided by $\mathrm{Yu}$ and Oh in Ref. [12] is therefore the simplest for $d=3$.

Proof. - The orthogonality graph of a SIC set has to obey at least the following necessary conditions: (a) that the graph has a $[3,1]$-representation, and $(b)$ that the graph has a fractional chromatic number greater than 3 .

From condition (a) it follows that the graph must be square free, because for a projector represented by a vertex of the square, the other two connected to it must be in the orthogonal plane, and the fourth is orthogonal to both, so it must be the same as the first.

The first step is to generate all nonisomorphic, i.e., not obtained via a relabeling, square-free connected graphs with 12 or fewer vertices and then calculate their chromatic number. It is sufficient to consider connected graphs since for a disconnected graph the chromatic number is the largest chromatic number of its connected components.

For this, we use the utility geng from the software package nauty v2.5r9 [42], and we find 143129 graphs with such properties. Among them, there is only one graph $G$ with $\chi(G)>3$, which is depicted in Fig. 1(c). 


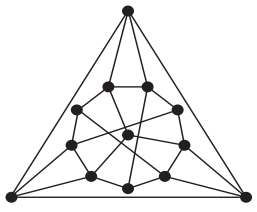

(a)

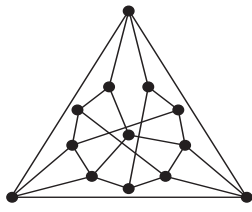

(b)

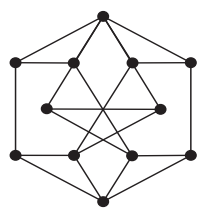

(c)
FIG. 1: (a) Yu-Oh graph $G_{\mathrm{YO}}$, (b) $G_{\mathrm{YO}}$ minus one edge. (c) The only square-free connected 12 -vertex graph with chromatic number $\chi(G)>3$.

By solving the linear program in Eq. (6) with exact arithmetic [43], one finds that its fractional chromatic number is $\chi_{f}(G)=3$.

One can go further and ask whether there are other SIC graphs with 13 vertices aside from the Yu-Oh graph $G_{\mathrm{YO}}$, depicted in Fig. 1(a). There are in total eight square-free graphs with 13 vertices and $\chi(G)>3$ [44], and out of these eight graphs, only three have $\chi_{f}(G)>3$ [45]. Two of them are depicted in Fig. 1, (a) $G_{\mathrm{YO}}$ and (b) $G_{\mathrm{YO}}$ minus one edge, together with the 12-vertex graph (c), which is a common induced subgraph of all remaining 13-vertex graphs with $\chi(G)>3$.

The existence of a representation in dimension $d=3$ for a given orthogonality graph can be written as a minimization of a polynomial function. In fact, the scalar product of two complex vectors can be written as a polynomial with vector entries as variables; hence, orthogonality conditions correspond to its zeros. A numerical search was not able to find a solution for such graphs in $d=3$.

Conclusion. - We have started arguing that the definition of "state-independent contextuality scenario" used in Ref. [35] is inconsistent with almost all of the previous literature on the topic and is not state independent on an operational level because the realization of the scenario depends on the state. Then we have shown that the criterion proposed in Ref. [35] does not solve the problem of whether or not a set of quantum tests reveals state-independent contextuality in the sense defined in most of the literature, including all experimental implementations and applications. Then we have presented a solution to this problem and explained the connection between this solution and the results in Ref. [35]. Finally, we have used our result to prove that the $\mathrm{Yu}-\mathrm{Oh}$ set is the simplest set of elementary quantum tests revealing state-independent contextuality in dimension three. Our results clarify the structure of state-independent contextuality and - as we demonstrated on an example - enable the systematic investigation of state-independent contextuality sets.

The authors thank P. Horodecki, J. R. Portillo, R. Ramanathan, and S. Severini for the useful conversations. This work was supported by Project No. FIS2011-29400 (MINECO, Spain) with FEDER funds, the FQXi large grant project "The Nature of Information in Sequential Quantum Measurements," and by the European Union (ERC Starting Grant No. GEDENTQOPT). Theorems 4 and 5 incorporate results that appeared in an unpublished article [34].

* Electronic address: adan@us.es

$\dagger$ Electronic address: matthias.kleinmann@uni-siegen.de

¥ Electronic address: costantino.budroni@uni-siegen.de

[1] M. Howard, J. J. Wallman, V. Veitch, and J. Emerson, Contextuality supplies the "magic" for quantum computation, Nature (London) 510, 351 (2014).

[2] R. Raussendorf, Contextuality in measurement-based quantum computation, Phys. Rev. A 88, 022322 (2013).

[3] N. Delfosse, P. A. Guerin, J. Bian, and R. Raussendorf, Wigner function negativity and contextuality in quantum computation on rebits, Phys. Rev. X 5, 021003 (2015).

[4] E. P. Specker, Die Logik nicht gleichzeitig entscheidbarer Aussagen, Dialectica 14, 239 (1960) [The logic of nonsimultaneously decidable propositions, arXiv:1103.4537].

[5] S. Kochen and E. P. Specker, The problem of hidden variables in quantum mechanics, J. Math. Mech. 17, 59 (1967).

[6] M. Pavičić, J.-P. Merlet, B. D. McKay, and N. D. Megill, Kochen-Specker vectors, J. Phys. A: Math. Gen. 38, 1577 (2005); 38, 3709 (2005).

[7] R. W. Spekkens, D. H. Buzacott, A. J. Keehn, Ben Toner, and G. J. Pryde, Preparation contextuality powers parity-oblivious multiplexing, Phys. Rev. Lett. 102, 010401 (2009).

[8] J. S. Bell, On the Einstein Podolsky Rosen Paradox, Physics (Long Island City, N.Y.) 1, 195 (1964).

[9] A. Cabello, Experimentally testable state-independent quantum contextuality, Phys. Rev. Lett. 101, 210401 (2008).

[10] P. Badziąg, I. Bengtsson, A. Cabello, and I. Pitowsky, Universality of state-independent violation of correlation inequalities for noncontextual theories, Phys. Rev. Lett. 103, 050401 (2009).

[11] X.-D. Yu and D. M. Tong, Coexistence of KochenSpecker inequalities and noncontextuality inequalities, Phys. Rev. A 89, 010101(R) (2014).

[12] S. Yu and C. H. Oh, State-independent proof of KochenSpecker theorem with 13 rays, Phys. Rev. Lett. 108, 030402 (2012).

[13] I. Bengtsson, K. Blanchfield, and A. Cabello, A KochenSpecker inequality from a SIC, Phys. Lett. A 376, 374 (2012).

[14] G. Kirchmair, F. Zähringer, R. Gerritsma, M. Kleinmann, O. Gühne, A. Cabello, R. Blatt, and C. F. Roos, State-independent experimental test of quantum contextuality, Nature (London) 460, 494 (2009).

[15] E. Amselem, M. Rådmark, M. Bourennane, and A. Cabello, State-independent quantum contextuality with single photons, Phys. Rev. Lett. 103, 160405 (2009).

[16] O. Moussa, C. A. Ryan, D. G. Cory, and R. Laflamme, Testing contextuality on quantum ensembles with one clean qubit, Phys. Rev. Lett. 104, 160501 (2010).

[17] C. Zu, Y.-X. Wang, D.-L. Deng, X.-Y. Chang, K. Liu, P.Y. Hou, H.-X. Yang, and L.-M. Duan, State-independent 
experimental test of quantum contextuality in an indivisible system, Phys. Rev. Lett. 109, 150401 (2012).

[18] V. D'Ambrosio, I. Herbauts, E. Amselem, E. Nagali, M. Bourennane, F. Sciarrino, and A. Cabello, Experimental implementation of Kochen-Specker set of quantum tests, Phys. Rev. X 3, 011012 (2013).

[19] G. Cañas, S. Etcheverry, E. S. Gómez, C. Saavedra, G. B. Xavier, G. Lima, and A. Cabello, Experimental implementation of an eight-dimensional KochenSpecker set and observation of its connection with the Greenberger-Horne-Zeilinger theorem, Phys. Rev. A 90, 012119 (2014).

[20] K. Horodecki, M. Horodecki, P. Horodecki, R. Horodecki, M. Pawłowski, and M. Bourennane, Contextuality offers device-independent security, arXiv:1006.0468.

[21] A. Cabello, Proposal for revealing quantum nonlocality via local contextuality, Phys. Rev. Lett. 104, 220401 (2010).

[22] L. Aolita, R. Gallego, A. Acín, A. Chiuri, G. Vallone, P. Mataloni, and A. Cabello, Fully nonlocal quantum correlations, Phys. Rev. A 85, 032107 (2012).

[23] O. Gühne, C. Budroni, A. Cabello, M. Kleinmann, and J.-A. Larsson, Bounding the quantum dimension with contextuality, Phys. Rev. A 89, 062107 (2014).

[24] G. Cañas, M. Arias, S. Etcheverry, E. S. Gómez, A. Cabello, G. B. Xavier, and G. Lima, Applying the simplest Kochen-Specker set for quantum information processing, Phys. Rev. Lett. 113, 090404 (2014).

[25] A. Peres, Incompatible results of quantum measurements, Phys. Lett. A 151, 107 (1990).

[26] N. D. Mermin, Simple unified form for the major nohidden-variables theorems, Phys. Rev. Lett. 65, 3373 (1990).

[27] A. Cabello, S. Severini, and A. Winter, Graph-theoretic approach to quantum correlations, Phys. Rev. Lett. 112, 040401 (2014).

[28] A. Acín, T. Fritz, A. Leverrier, and A. B. Sainz, A combinatorial approach to nonlocality and contextuality, Commun. Math. Phys. 334, 533 (2015).

[29] A. Cabello, Simple explanation of the quantum violation of a fundamental inequality, Phys. Rev. Lett. 110, 060402 (2013).

[30] T. Fritz, A. B. Sainz, R. Augusiak, J. Bohr Brask, R. Chaves, A. Leverrier, and A. Acín, Local orthogonality: A multipartite principle for correlations, Nat. Commun.
4, 2263 (2013).

[31] B. Yan, Quantum correlations are tightly bound by the exclusivity principle, Phys. Rev. Lett. 110, 260406 (2013).

[32] A. B. Sainz, T. Fritz, R. Augusiak, J. Bohr Brask, R. Chaves, A. Leverrier, and A. Acín, Exploring the local orthogonality principle, Phys. Rev. A 89, 032117 (2014).

[33] A. Cabello, Exclusivity principle and the quantum bound of the Bell inequality, Phys. Rev. A 90, 062125 (2014).

[34] A. Cabello, State-independent quantum contextuality and maximum nonlocality, arXiv:1112.5149v1.

[35] R. Ramanathan and P. Horodecki, Necessary and sufficient condition for state-independent contextual measurement scenarios, Phys. Rev. Lett. 112, 040404 (2014).

[36] P. Kurzyński and D. Kaszlikowski, Contextuality of almost all qutrit states can be revealed with nine observables, Phys. Rev. A 86, 042125 (2012).

[37] A. A. Klyachko, M. A. Can, S. Binicioğlu, and A. S. Shumovsky, Simple test for hidden variables in spin-1 systems, Phys. Rev. Lett. 101, 020403 (2008).

[38] E. R. Scheinerman and D. H. Ullman, Fractional Graph Theory: A Rational Approach to the Theory of Graphs (Dover, New York, 2011).

[39] M. Kleinmann, C. Budroni, J.-Å. Larsson, O. Gühne, and A. Cabello, Optimal inequalities for state-independent contextuality, Phys. Rev. Lett. 109, 250402 (2012).

[40] R. A. Horn and C. R. Johnson, Matrix Analysis (Cambridge University Press, New York, 1985).

[41] L. Vandenberghe and S. Boyd, Semidefinite programming, SIAM Rev. 38, 49 (1996).

[42] B. D. McKay and A. Piperno, Practical graph isomorphism, II, J. Symb. Comput. 60, 94 (2014).

[43] K. Fukuda, software library cddlib v094g, http://www. ifor.math.ethz.ch/ fukuda/cdd_home/

[44] In the graph6 encoding (http://cs.anu.edu.au/ ebdm/ data/formats.html), they are: "L?AEB?oDDIQSUS", "L?AEB? oFDHISPS", "L?ABA_OO_iREJa", "L?ABAagF@bWgHc", "L?ABEagE'gH" "c" (which is G minus one edge), "L?AB?vOLDPHa'o" (which is $G_{Y O}$ ), "L?BDA_gEREHcac, and "L?'D@bCUCbDgWc".

[45] In the graph6 encoding, "L?ABEagE' $g H^{\prime}$ " c $"$ ", having $\chi_{f}=$ $19 / 6$, "L?AB?vOLDPHa'o", having $\chi_{f}=35 / 11$, and "L?'D@bCUCbDgWc", having $\chi_{f}=13 / 4$. 